PROCEEDINGS OF THE

AMERICAN MATHEMATICAL SOCIETY

Volume 35, Number 2, October 1972

\title{
TANGENT BUNDLES OF HOMOGENEOUS SPACES ARE HOMOGENEOUS SPACES
}

\author{
R. W. BROCKETT AND H. J. SUSSMANN
}

ABSTRACT. In this paper we describe how the tangent bundle of a homogeneous space can be viewed as a homogeneous space.

The purpose of this note is to establish a simple result on the structure of the tangent bundle of a homogeneous space. Even though it is both natural and elementary it does not appear to be in the literature.

We shall associate with every Lie group $G$ another Lie group $G^{*}$, constructed as a semidirect product of $G$ with the Lie algebra of $G$ (the precise definition is given below).

Our result is:

THEOREM. If a Lie group $G$ acts transitively and with maximal rank on a differentiable manifold $X$, then $G^{*}$ acts transitively and with maximal rank on the tangent bundle of $X$.

Clearly, our result implies that the tangent bundle of a coset space $G / H$ is again a coset space and moreover, is of the form $G^{*} / K$ for some closed subgroup $K$ of $G^{*}$. We will compute $K$ below.

We now define $G^{*}$ and prove the theorem. Let $L$ be the Lie algebra of $G$, thought of as the tangent space of $G$ at the identity. For each $g \in G$, we let $a d(g)$ denote the differential at the identity of the inner automorphism $x \rightarrow g x g^{-1}$ of $G$. Thus $a d$ is a (not necessarily one-to-one) homomorphism of $G$ into the group of linear automorphisms of $L$. We define $G^{*}$ as the product manifold $L \times G$, with the group operation given by

$$
(a, g) \cdot\left(a^{\prime}, g^{\prime}\right)=\left(a+a d(g)\left(a^{\prime}\right), g g^{\prime}\right) .
$$

The verification that $G^{*}$ is a group is trivial and will be omitted. Also, it is clear that the operation defined by (1) is differentiable, so that $G^{*}$ is a Lie group.

Now, let $G$ act differentiably on a manifold $X$. For each $x \in X$, let $\theta_{x}: G \rightarrow X$ be defined by $\theta_{x}(g)=g x$.

If $x \in X$, then the differential of $\theta_{x}$ at the identity maps $L$ into $X_{x}$ (the tangent space of $X$ at $x$ ). If $a \in L$, we let $\bar{a}(x)=d \theta_{x}(a)$. It is easy to see that $\bar{a}$ is a smooth vector field on $X$. Use $\tau(X)$ to denote the tangent bundle of $X$.

Received by the editors November 24, 1971.

AMS 1970 subject classifications. Primary 53C30, 57E25.

Key words and phrases. Tangent bundles, homogeneous spaces.

(c) American Mathematical Society 1972 
We define a left action of $G^{*}$ on $\tau(X)$ by

$$
(a, g) \cdot v=d \sigma_{g}(v)+\bar{a}(g \cdot \pi(v)) \text { for } v \in \tau(X) .
$$

Here $\pi$ denotes the natural projection from $\tau(X)$ onto $X$ (i.e. $\pi(v)=x$ if and only if $\left.v \in X_{x}\right)$ and $\sigma_{g}: X \rightarrow X$ is the map $x \rightarrow g x$. Clearly, both $d \sigma_{g}(v)$ and $\bar{a}(g \cdot \pi(v))$ belong to $X_{g \cdot \pi(v)}$, so that the sum is defined. We omit the trivial verification that $(2)$ satisfies

and

$$
(0, e) \cdot v=v \quad \text { for all } v \in \tau(X)
$$

$$
\left((a, g) \cdot\left(a^{\prime}, g^{\prime}\right)\right) \cdot v=(a, g) \cdot\left(\left(a^{\prime}, g^{\prime}\right) \cdot v\right) .
$$

Also, it is clear that the action of $G^{*}$ on $\tau(X)$ defined by (2) is differentiable.

Now assume that $G$ acts transitively and with maximal rank on $X$. If $v$ and $v^{\prime}$ belong to $\tau(X)$, then there exists $g \in G$ such that $g \cdot \pi(v)=\pi\left(v^{\prime}\right)$ (by the transitivity). Since $G$ acts with maximal rank, there is an $a \in L$ such that $d \theta_{\pi\left(v^{\prime}\right)}(a)=v^{\prime}-d \sigma_{g}(v)$.

Therefore $(a, g) \cdot v=v^{\prime}$. This shows that $G^{*}$ acts transitively on $\tau(X)$. We now show that $G^{*}$ acts with maximal rank. Let $G_{0}$ be the connected component of the identity element of $G$. Then $G_{0}$ acts with maximal rank on $X$. Therefore the $G_{0}$-orbits are open submanifolds of $X$. If $Y$ is a $G_{0^{-}}$ orbit, then $G_{0}$ acts transitively and with maximal rank on $Y$. We have already shown that this implies that $G_{0}^{*}$ acts transitively on $\tau(Y)$. Since $G_{0}^{*}$ is obviously connected, it follows that $G_{0}^{*}$ acts with maximal rank on $\tau(Y)$. Now $\tau(X)$ is obviously the union of the sets $\tau(Y)$, where $Y$ is a $G_{0^{-}}$ orbit in $X$. These sets are open submanifolds of $\tau(X)$. It follows that $G_{0}^{*}$ acts with maximal rank on $\tau(X)$. Then, necessarily, $G^{*}$ also acts with maximal rank on $\tau(X)$.

RemarKs. (A) If $G$ acts transitively on $X$ it does not follow that $G^{*}$ acts transitively on $\tau(X)$ (let $X=$ the real line with its usual one-dimensional differentiable structure and $G$-the real line considered as a discrete group).

(B) If $H$ is a closed subgroup of $G$, then $H^{*}$ can be identified, in an obvious way, with a closed subgroup of $G^{*}$. One verifies easily that the isotropy group of $0\left(\varepsilon X_{x}\right)$ corresponding to the action of $G^{*}$ on $\tau(X)$ is precisely $H_{x}^{*}$, where $H_{x}$ is the isotropy group of $x$ corresponding to the action of $G$ on $X$. In particular, we have the diffeomorphism $\tau(G / H) \simeq$ $G^{*} / H^{*}$.

Aiken Computation laboratory, Harvard University, Cambridge, MasSACHUSETTS 02138 (Current address of R. W. Brockett)

Department of Mathematics, University of Chicago, Chicago, Illinois 60637

Current address (H. J. Sussmann): Department of Mathematics, Rutgers University, New Brunswick, New Jersey 08903 